\title{
émulations
}

\section{Céder sa place. Logique situationnelle et catégorisation dans le métro de Saint-Pétersbourg}

\author{
Victoria Chantseva
}

Émulations - Revue de sciences sociales 2021, Varia, En ligne.

Article disponible à l'adresse suivante

https://ojs.uclouvain.be/index.php/emulations/article/view/chantseva_varia

\section{Pour citer cet article}

Victoria Chantseva, «Céder sa place. Logique situationnelle et catégorisation dans le métro de Saint-Pétersbourg », Émulations, Varia, En ligne. Mise en ligne le 14 octobre 2021. DOI : 10.14428/emulations.varia.033

Distribution électronique : Université catholique de Louvain (Belgique) : ojs.uclouvain.be

(C) Cet article est mis à disposition selon les termes de la Licence Creative Commons Attribution, Pas d'Utilisation Commerciale 4.0 International. http://creativecommons.org/licenses/by-nc/4.0/

Éditeur : Émulations - Revue de sciences sociales / Presses universitaires de Louvain https://ojs.uclouvain.be/index.php/emulations

ISSN électronique : 1784-5734

PUL PRESSES UNIVERSITAIRES 


\title{
Céder sa place. \\ Logique situationnelle et catégorisation dans le métro de Saint-Pétersbourg
}

Victoria Chantseva $^{1}$

\begin{abstract}
[Résumé] : L'article s'intéresse aux significations des gestes de politesse dans les transports en commun en s'appuyant sur une ethnographie du métro de Saint-Pétersbourg. Des séances d'observation ainsi que des descriptions que les usagers de métro donnent de leur expérience quotidienne - notamment celle liée au fait de céder sa place à l'autre - font émerger les éléments d'une socialisation différenciée selon le genre. Les mêmes gestes peuvent s'inscrire dans les logiques d'action différentes, selon l'âge et le sexe des personnes engagées. Toutefois, les connotations genrées ou celles liées à la hiérarchie des âges peuvent faire l'objet de contestations. Céder la place pour se montrer courtois avec les femmes ou pour faire preuve de respect envers les personnes âgées n'est pas toujours accepté car cela tend à instaurer des rapports soulignant la différence entre les personnes impliquées. C'est ce que les acteurs essayent de surmonter en recourant à des tactiques de précaution qui rendent le geste acceptable.
\end{abstract}

Mots clés : âge, civilité urbaine, genre, transports publics.

[Abstract]: Drawing on an ethnographic fieldwork, this article examines the meanings attached to polite behaviors in Saint Petersburg subway. Participant observation, as well as interviews, focusing on daily experiences of subway - in particular those related to offering one's seat to another person - reveal gender-differentiated socialization. The same practice can have different justifications depending on the age and gender of the actors involved. However, the connotations of gender or age hierarchy can be contested. Offering one's seat to be gallant to women or to show respect to elderly people is not always acceptable because the practice implies differences of status. That is why actors use precautionary tactics that make the interaction acceptable.

Key-words: age, gender, public transports, urban manners.

La conformité aux attentes de civilité semble revêtir une grande importance pour les contacts anonymes dans les espaces publics. Plusieurs recherches ont mis en évidence les règles de convenances plus ou moins tacites auxquelles obéissent les rencontres entre inconnus dans les contextes urbains (Birenbaum, Sagarin, 1973; Goffman, 1963; 1973 [1956] ; 1974 [1967] ; Lofland, 1973 ; 1998). Prolifiques, contradictoires, demandant un effort interprétatif, ces règles ne constituent pourtant pas un code de conduite univoque qu'il suffirait de mettre mécaniquement en œuvre. Les situations auxquelles

\footnotetext{
${ }^{1}$ Université Sorbonne Paris Nord, EXPERICE, France.
} 
celui-ci s'applique sont d'une variabilité prodigieuse donnant matière à des malentendus et à des erreurs de jugement. L'ordre de l'interaction publique est fragile (Cefaï, 2013) et lorsque les interactants ne s'accordent pas sur le contenu et la portée des règles ou bien sur le sens des gestes accomplis, les situations banales peuvent facilement basculer vers des troubles (Brevigleri, Trom, 2003), voire des conflits et des « disputes de politesse» (Gayet-Viaud, 2008b).

Une des difficultés de ce type d'interactions tient à la tension inhérente entre les deux principes qui les gouvernent. D'une part, l'exigence de la neutralité démocratique, liée au fait qu'il s'agit de rapports entre quidams, entre individus anonymes, égaux et libres de toutes caractéristiques biographiques (Sennet, 1979 [1977]). Dans un certain sens, la règle de «l'inattention civile » - le fait d'éviter de s'intéresser trop à l'autre en préservant ses distances, constituant, selon Erving Goffman (1963: 84), la base de la politesse urbaine - renvoie au principe de la neutralité égalitaire des coprésences anonymes. En correspondance avec ce principe, certaines recherches analysent les interactions entre les passants en appréhendant ces derniers en tant que figurants impersonnels et substituables, sorte d'unités d'action, habiles ou malhabiles dans l'arrangement de la mobilité ou de l'occupation d'espaces selon les modalités prévisibles pour tous les protagonistes (Ryave, Schenkein, 1974; Weilenmann, Normark, Laurier, 2014; Wolf, 1973²).

D'autre part, les interactions en question impliquent une catégorisation des partenaires, c'est-à-dire l'identification de l'appartenance des individus à différents groupes (d'âge, d'ethnie, de profession, de sexe, etc.). « The world of strangers » (Lofland, 1973) se révèle the world of identities:

J'affirme [/ shall argue] que la situation d'un «pur » anonymat serait intolérable.

J'affirme que les humains ne peuvent vivre dans les villes que parce qu'ils en savent beaucoup sur les inconnus [strangers] les entourant. Et j'affirme que ce savoir - la condition sine qua non de la vie urbaine - est lui-même possible grâce à un « ordonnancement » de la population de la ville de sorte que les apparences et les positions dans l'espace fournissent des indices relativement fiables sur les «identités ». [lbid. : $x$, traduction de l'auteure]

Plusieurs auteurs thématisent ce travail de catégorisation en le plaçant au cœur de la régulation des conduites, au même titre que le travail d'ajustement aux contraintes physiques des équipements urbains (Lee, Watson, 1992; Quéré, Brezger, 1992; Sacks, 1979; Sudnow, 1972). Certaines ethnographies se focalisent ainsi sur la vie des quartiers ethniques (Duneier, 1999; Liebow, 2011 [1967]) ou bien sur la présence publique des individus appartenant aux catégories vulnérables, notamment des femmes, jeunes ou personnes racisées. La focalisation sur ces groupes conduit à mettre l'accent sur les

2 Ce rapport formaliste conduit parfois à des assertions manifestement réductionnistes: "Généralement, les piétons adultes semblent traiter les enfants de sept ans ou moins comme des "bagages". » (Wolff, $1973: 45)$ 
discriminations subies, la catégorisation basculant vers une manifestation violente des hiérarchies sociales (Anderson, 1990; Gardner, 1995; Nielsen, 2004).

Une troisième approche consiste à s'attarder sur la tension même entre l'anonymat des passants et leur catégorisation mutuelle, en décortiquant, à partir de situations concrètes, l'oscillation des interactions entre le registre de parité et celui de différenciation (Joseph, 1984; 2007; Gayet-Viaud, 2008a ; 2009; 2011; Tavory, 2011). L'accent est mis ici sur le fait que la « bonne mesure » de la civilité est celle qui évite les deux écueils : l'indifférence, d'une part, et l'ingérence invasive, d'autre part (Bidet et al., 2015; Gayet-Viaud, 2019). Le présent article s'inscrit dans cette dernière perspective. À la différence des recherches focalisées sur les stratégies d'évitement des contacts dans le métro (Levine, Vinson, Wood, 1973), cette étude se penche sur une pratique qui suppose l'entrée en contact. Il s'agit de s'intéresser au sens du geste de l'offre de sa place dans les transports publics en pointant sa dimension problématique: selon quels critères les acteurs évaluent-ils la nécessité de rompre avec la règle "d'inattention civile » en proposant à l'autre sa place?

Pour établir ce que les usagers de métro prennent en compte pour s'engager (ou non) dans l'action en question, nous allons nous appuyer sur une enquête conduite dans le métro de Saint-Pétersbourg33, où certaines normes informelles de bienséance entrent en symbiose avec les obligations institutionnelles. En effet, dans le métro de Saint-Pétersbourg, certaines catégories de passagers (personnes âgées, personnes handicapées, femmes enceintes et passagers avec enfants) ont le droit formel de s'asseoir en priorité. L'accès aux sièges est donc régulé non seulement par le principe du «premier arrivé, premier servi » (Schwartz, 1975), mais aussi par l'appartenance de certains voyageurs à des catégories prioritaires. Stipulée par les annonces sonores et les panneaux affichés dans les rames, cette règle d'usage n'est pourtant pas réellement contraignante, aucune pénalisation officielle n'étant prévue en cas de transgression ${ }^{4}$. En même temps, héritée de l'époque soviétique, une certaine culture des débats en public, axée sur la dénonciation et la culpabilisation des individus (Vakhtin, Firsov, 2017; Kelly, 2001), les fait glisser facilement dans le registre du dénigrement et du jet de l'opprobre, ce qui laisse planer sur toute transgression des règles de bienséance la menace d'être violemment critiquée.

\footnotetext{
${ }^{3}$ Située au bord de la mer Baltique, Saint-Pétersbourg est, après Moscou, la deuxième grande ville de Russie par nombre d'habitants. Du fait de son histoire spécifique, cette ancienne capitale est considérée comme étant la plus proche de la civilisation occidentale, tant par la structure de son économie et de ses institutions, que par ses mœurs, réputées être les plus policées du pays. L'un des plus profonds en Europe, le métro pétersbourgeois compte 5 lignes et 64 stations au moment de l'enquête. Elles sont toutes souterraines, avec des trajets entre les stations allant de 3 à 8 minutes.

${ }^{4}$ Dans son article consacré aux sentiments de confiance dans le métro new yorkais, Stéphane Tonnelat (2012) note également que, de fait, plusieurs règles du métro échappent au contrôle des services d'ordre et sont sujettes à des négociations, voire à des infractions fréquentes.
} 
L'article se donne pour objectif de mettre en lumière les attentes implicites liées à l'occupation des places assises, de comprendre les appuis normatifs des microinteractions autour de celles-ci et de saisir les types de compétences mises en œuvre. Sans prétendre fournir un inventaire complet de ces éléments, il s'agit de cerner ce qui entre en jeu dans ces situations concrètes, dès l'apprentissage même. La perspective interactionniste est adoptée afin de rendre compte des enjeux spécifiques des conduites dans l'espace public et des stratégies de " coprésence des sexes» (Goffman, 2002 [1977]) et des âges.

Les données viennent d'une enquête ethnographique effectuée en 2012-2013. Elle comporte, d'abord, des séances d'observation participante: en m'installant dans une rame de métro au même titre que les autres voyageurs, j'ai fixé dans mon carnet les déplacements des passagers, les postures, les échanges verbaux et non verbaux. Sur une période allant de janvier à décembre 2012, 30 séances d'observation de deux à quatre heures ont été effectuées. L'ensemble des lignes de métro de Saint-Pétersbourg a été progressivement investi, à des horaires variés (heures de pointe le matin et le soir, heures creuses de début d'après-midi). Le continuum temporel, capté ainsi sur le papier, était ensuite découpé en séquences, très courtes pour la plupart d'entre elles, portant sur l'action de s'asseoir et/ou de céder sa place. La deuxième partie des données, plus large, est constituée de 50 entretiens semi-directifs conduits auprès d'hommes et de femmes de différents âges et de catégories professionnelles variables, quoique relativement homogènes, qui prennent le métro plus ou moins fréquemment5. L'objectif premier de ces entretiens était de faire émerger les façons dont les personnes se perçoivent et les manières dont elles justifient leurs comportements au cours des déplacements. Il s'agissait aussi de les inciter à raconter les histoires tirées de leur propre expérience. Ces récits ont constitué - à côté des séquences venant de l'observation - la matière première de l'analyse qui suit.

\footnotetext{
${ }^{5} \mathrm{Au}$ vu de l'impossibilité pratique de solliciter les personnes observées sur-le-champ, les entretiens ont été réalisés avec des personnes recrutées par principe de "boule de neige 》, en visant, notamment, la diversité des âges et la parité des sexes ( 27 femmes et 23 hommes). À l'exception d'une concierge, d'un mécanicien et d'un ouvrier, l'échantillon représente la classe moyenne urbaine, à haut niveau de diplôme $(\mathrm{Bac}+5$ pour la plupart, sinon en cours du cursus académique) et au niveau économique modeste (plusieurs des interviewés ont déclaré toucher un salaire inférieur à la moyenne ou ne pas avoir des revenus stables). En raison de l'échantillonnage qualitatif et de la portée restreinte des entretiens, se limitant à l'expérience des transports en commun, sans entrer dans les détails du parcours de vie, nous ne pourrons pas invoquer les caractéristiques socio-économiques des enquêtés comme facteurs explicatifs des comportements.
} 


\section{Ne pas exprimer publiquement l'envie de s'asseoir, une exigence genrée?}

Les bonnes manières, remarque Goffman (1963: 61), consistent souvent dans la réserve devant les différentes formes de biens. Par exemple, il est de bon ton de manger comme si le fait de se nourrir n'était pas très important. D'après les témoignages des usagers du métro, la place assise est justement cette chose pour laquelle il est mal vu d'exprimer une convoitise ou encore de montrer qu'on lui accorde de la valeur ${ }^{6}$. Ce sont donc les gestes frénétiques et l'agitation excessive que les voyageurs perçoivent comme des manquements aux bonnes manières et qui trahissent soit un jeune âge, soit - comme on peut le voir dans le récit suivant - l'absence de familiarité avec la vie dans une grande ville:

J'ai remarqué ces deux femmes [...] elles, en montant dans la rame, il n'y avait pas beaucoup de monde, mais elles se frayaient activement un passage, se précipitaient vers les banquettes en bousculant les autres et en criant... ben, l'une d'elles s'est avancée en criant : « Manya, Manya, viens, je te garde une place ici ! ». Eh bien, j'en ai conclu qu'elles venaient d'un village, parce que, bon... on ne se conduit pas comme ça, les adultes, en ville, on ne court pas à travers la rame en criant « Manya, viens ici, je te garde une place », parce que seuls les enfants se comportent comme ça. Eh bien... les enfants ils courent parfois en bousculant les autres et en criant « Maman, maman, viens ici, je te garde une place ! ». [Olga, employée de musée, 24 ans]

La définition des deux femmes comme villageoises et la mise en parallèle de leur conduite avec celle des enfants s'appuient sur la valorisation de la retenue devant les avantages d'une place assise. Ces remarques révèlent la règle implicite que tout bon citoyen se doit de respecter: contrôler ses élans et ne pas faire grand cas d'une place assise dans les transports. D'où l'interdit informel des gestes d'appropriation. On peut supposer donc que la désapprobation de tels gestes vient tout simplement du fait que, s'autorisant à empêcher les autres d'accéder à ces biens, on se conduit en égoïste: on usurpe des biens censés être pour tous. On peut trouver l'expression de cette idée dans la séquence suivante:

Parmi les passagers qui montent se trouve une fille d'une dizaine d'années. À pas vif, elle se dirige droit vers les places libres au milieu d'une banquette, s'assoit juste au milieu en mettant la main sur une place à côté. Une femme d'une cinquantaine d'années (sa grand-mère ?) la suit, s'arrête devant elle et dit d'un ton ironique : « Et pourquoi ne veux-tu pas te coucher ici en plus? ». La fille retire la main, se cale de façon plus compacte. La femme s'assoit à côté de la fille qui répond d'un ton plaintif : «C'est que je voulais garder la place pour toi ». La femme répond: « Luba, on ne fait pas des choses comme ça. Moi, je... eh ben... il se peut que quelqu'un d'autre veuille

${ }^{6}$ Marc Augé note à cet égard que les habitués du métro parisien se reconnaissent à « l'économie élégante » et à " l'extrême précision » des gestes, la manière discrète et ajustée dont on s'assoit étant une preuve indéniable d'expérience (2009 : 14-15). 
s'asseoir aussi... Je te donne le livre ? ». Elle sort un livre, le donne à la fille qui se met

à le feuilleter. [Journal de terrain, 10/12/12, Ligne 3]

La désapprobation du geste de réserver la place pour quelqu'un d'autre, qui apparaissait déjà en creux dans le discours d'Olga, trouve ici une expression plus nette. L'absence de référence au genre, le « on » (" on ne fait pas des choses comme ça ») auquel se réfère la femme, tout comme le « on » d'Olga marquent ici la dimension universelle de la conduite civile, l'étalon pour tous les citadins: «on ne se conduit pas comme ça, les adultes, en ville ${ }^{7}$ ».

Or, si l'on creuse davantage, on découvre que le devoir de désintéressement devant les places assises ne s'applique pas de la même manière chez les hommes que chez les femmes. Pour les hommes, le seul fait de se soucier ouvertement de choses telles qu'un siège dans les transports paraît particulièrement incongru. Ceci peut s'observer, d'abord, dans les dénigrements de ceux et surtout de celles qui s'empressent vers les sièges. L'idée même de chercher à s'installer sur un siège est désapprouvée moralement par les hommes. C'est le cas de ce traducteur de 25 ans, qui fait une description caricaturale des «mémés roublardes»: elles «mesurent où précisément le bus va s'arrêter, elles se jettent en avant, les portes ne sont pas encore ouvertes, mais elles sont déjà là et poussent donc avec les coudes, arrivent aux sièges et prennent les quatre à la fois. »

En revanche, les femmes interviewées se montrent plus attachées au confort du voyage. Pour certaines d'elles, l'envie de voyager assises semble tout à fait légitime à condition de ne pas être trop affichée. La confrontation des deux discours suivants le met en évidence. En expliquant pourquoi elle s'assoit systématiquement en voyant une place libre, Svetlana, une étudiante de 18 ans, déclare sentir «toujours une certaine fatigue même si [elle] étai[t] assise dans la journée, disons, pendant deux ou trois cours [à l’Université]». Boris, un musicien de 30 ans, affirme, quant à lui: «Si j'étais assis pendant longtemps au travail et même si je me sens fatigué, je vais plutôt me tenir debout parce que j'ai une arrière-pensée: toujours assis, j'aurai un gros cul et pour un homme ce n'est pas beau ».

Nous voyons ici un rapport différencié à la fatigue en tant que justification de l'envie de s'asseoir dans les transports publics. Malgré son état physique, Boris déclare préférer rester debout afin de ne pas avoir « un gros cul ». Or, les femmes, plus exposées à la pression de la norme de la minceur (Darmon, 2003), n'ont jamais, elles, fait état de préoccupations liées au fait qu'une demi-heure assise dans le métro puisse les faire grossir. Svetlana - on peut supposer qu'à 18 ans le problème de l'âge comme source de fatigue ne se pose pas pour elle-s'exprime très différemment au sujet d'une situation

\footnotetext{
${ }^{7}$ Notons, pour la justesse grammaticale, que les deux phrases ont été prononcées en russe où le pronom neutre de la troisième personne n'existe pas. Cependant, dans la version originale, il n'y avait effectivement pas de marquage de genre. En traduisant au pied de la lettre, Olga dit : « nous ne courons pas comme ça », et la femme de la séquence dit : "les choses comme ça ne se font pas ».
} 
proche: en sentant «une certaine fatigue», elle préfère s'asseoir pour "piquer un somme, voilà, un peu, c'est normal ». À la différence de l'homme, la femme peut donc se permettre de prendre en compte sa fatigue, d'appréhender comme justifiée son envie de s'asseoir, et au-delà, de ne pas se sentir obligée de céder sa place à quelqu'un d'autre. Dans la partie suivante, nous verrons se préciser ces attentes différenciées à l'usage social du corps (Boltanski, 1971) qui contribuent à brouiller la neutralité de l'espace public.

\section{Se tenir debout : une posture d'homme}

Dans le métro pétersbourgeois, l'impératif d'endurance ne s'impose pas avec la même force aux femmes qu'aux hommes. Ceci devient encore plus explicite lorsqu'il s'agit de se tenir droit et debout ${ }^{8}$. Au-delà de la question de la réserve, c'est à cette exigence de verticalité que semble s'articuler le devoir des hommes de céder la place. Les manquements à cette prescription sont interprétés comme une dérogation patente à l'organisation spatiale. Comme l'a formulé une agente comptable de 41 ans : «Personne ne cède la place [...] Ça saute aux yeux, oui. Surtout quand on prend le métro et qu'on voit les hommes assis». Dans ce discours, si «ça saute aux yeux» que "personne ne cède la place », c'est parce que « les hommes sont assis ». Ce raisonnement fait référence à « une micro-configuration spatiale » (Goffman, 1977:37) où les positions des individus dans l'espace sont représentatives de leurs positions sociales relatives.

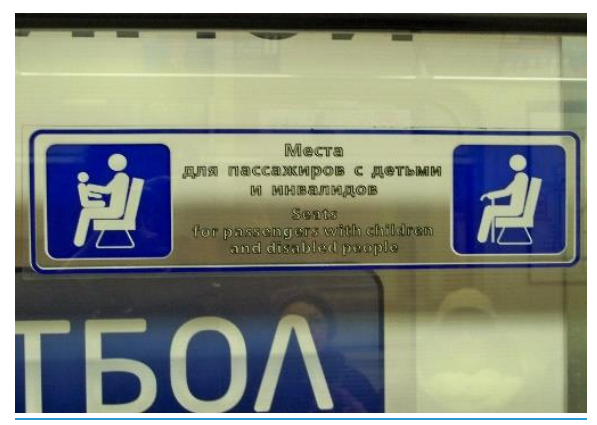

Photo 1. La signalétique «Places pour PASSAGERS AVEC ENFANTS ET PERSONNES HANDICAPÉES » (CHANTSEVA, 2013).

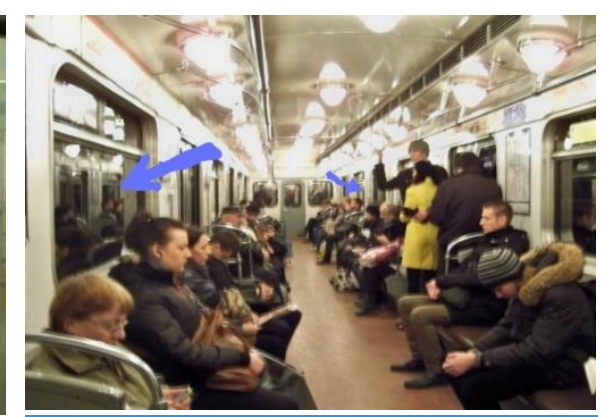

PHOTO 2. LA DISPOSITION DES SIGNALÉTIQUES DANS LE WAGON (CHANTSEVA, 2013).

\footnotetext{
${ }^{8}$ Selon Pierre Bourdieu, la posture droite est traditionnellement associée au masculin en tant qu'une « attestation de droiture » à la fois physique et morale (Bourdieu, $1998: 33$ ).
} 
Le fait que les hommes soient debout est perçu comme normal, c'est donc à eux de céder en premier leur place:

Arrivée à la station «Ploshchad' Alexandra Nevskogo », l'annonce passe: «Chers passagers, merci de céder la place aux femmes enceintes, personnes âgées et passagers avec enfants ». En face de moi, une femme de cinquante ans environ s'adresse tout de suite à un homme d'une trentaine d'années assis devant elle et tenant un sac à dos sur les genoux : « Vous avez entendu ? Ça concerne les hommes ». L'homme se lève, son visage exprime un mélange de confusion et d'étonnement. La femme continue de parler, je ne peux pas discerner les mots qui se fondent dans le bruit. Elle s'assoit en finissant son monologue à la cantonade : « Les hommes décents se lèvent toujours devant une dame ». La rame repart, l'homme reste debout en face de la femme, bloqué par les autres voyageurs. À la station suivante, I'homme au sacà-dos sort. [Journal de terrain, 08/11/12, Ligne 3]

La singularité du métro de Saint-Pétersbourg se manifeste dans cette séquence: grâce aux annonces sonores qui passent de manière très régulière ainsi qu'à la signalétique au-dessus des banquettes dans les wagons (photos 1 et 2), les passagers ont la possibilité de formuler leur demande de place en se référant à l'évidence des règles visualisées. Le deuxième aspect saillant est l'assurance avec laquelle la femme affirme que "ça concerne les hommes ", la précision ne figurant pourtant pas dans l'annonce à laquelle elle se réfère. C'est un « ça va de soi » qui s'entend dans sa réplique, indice de la « doxa de sexe » (Haicault, 1993: 8) en action, tandis que la référence à la décence enfonce le clou du jugement négatif à l'égard de l'homme. Nous voyons ici une irruption explicite des normes du genre dans les figures de justification des attentes liées à la distribution des places. Or, l'étonnement de l'homme au sac-à-dos suggère que le rappel à l'ordre qui le force à se lever n'est pas complètement légitime. Sous-entendant le bon droit de la femme non seulement de s'asseoir, mais aussi de punir le fautif par une réprimande, ce ton accusateur nie la neutralité de l'espace public où tout le monde aurait le droit de s'asseoir. De plus, la façon péremptoire dont la place assise est ici réquisitionnée enlève toute possibilité d'inscrire le geste dans le registre du don, l'homme étant obligé de se lever non pas par politesse, mais parce qu'on lui a fait honte?.

La compréhension de ce type de commentaires publics nécessite la prise en compte non seulement des modèles "soviétiques" de la désapprobation affichée devant tout le monde, mais aussi le fait qu'en Russie, les hiérarchies du genre sont traditionnellement fortes et visibles, et ceci malgré certains acquis égalitaires dans les politiques publiques, tels que l'accès des femmes au travail salarié, le droit au divorce, etc. (Zdravomyslova, Rotkirch, Temkina, 2009). Il est attendu des hommes et des femmes de jouer des rôles différents aussi bien dans l'espace public que privé et les variations de ces écarts selon la classe sociale sont plus faibles qu'en Europe de l'Ouest (Carlbäck, Gradskova, Kravchenko, 2012). Ainsi, d'après certains discours recueillis,

${ }^{9}$ Sur les impasses de la politesse extorquée, voir Gayet-Viaud (2008b ; 2011). 
céder sa place incombe en premier lieu aux hommes qui doivent le faire non pas parce que tout le monde le fait, mais parce qu'ils sont des hommes:

Oui, les hommes doivent céder la place! [...] Ils sont le sexe fort. Ils sont les défenseurs. Puisqu'ils ont gagné la guerre ${ }^{10}$, ils doivent céder la place aux femmes. [Sofia, femme au foyer, 31 ans]

Anna : S'il s'agit d'un militaire ou d'un marin, alors ça, je ne comprends pas du tout comment il peut être assis devant moi. [...]

Moi : Mais s'il se sent mal ?

Anna: Eh bien, je ne pense pas qu'il se sente mal, on a des guerriers malades ou quoi ? [...] Il doit avoir ça dans son sang qu'il faut céder la place à la femme ! C'est pourquoi je dis qu'aujourd'hui c'est la féminisation et les hommes sont très faibles, de sorte qu'ils sont souvent infirmes et malades. [Anna, enseignante dans le secondaire, 62 ans]

Le devoir de céder la place s'ancre dans une définition de l'homme comme « sexe fort ». Le propos de Sofia le laisse entendre de manière très lapidaire: les hommes doivent le faire parce qu'ils sont les « défenseurs ». La référence au devoir du soldat fonctionne comme une preuve du fait que les hommes sont bien le « sexe fort » et en comparaison avec l'activité militaire, laisser sa place à la femme apparaît comme un geste banal. La même allusion aux métiers militaires prend, chez Anna, des proportions encore plus naturalisantes. La conformité aux attentes sociales apparaît comme devant être infusée dans la chair, ce qui fait que le devoir moral est assimilé à une sorte de destin anatomique.

Cependant, il est important de souligner que, dans les faits, l'homme est plus tenu de se montrer fort que de l'être véritablement: personne, en général, ne conteste le fait qu'un homme puisse être fatigué ou souffrant. Ainsi, quand les voyageurs se connaissent (un couple, par exemple), la distribution des positions dans l'espace est beaucoup moins rigide, et ce n'est pas rare que les femmes cèdent la place à leurs compagnons ou restent debout alors que ces derniers sont assis. Or, dans les interactions entre les inconnus, il s'agit surtout de jouer sur les apparences en «maîtrisant l'impression » (Goffman, 1973:197), car personne n'a ni le temps ni l'intérêt pour la réalité des expériences personnelles. C'est pourquoi, s'ils veulent rester assis dans une rame remplie, les passagers masculins peuvent se sentir contraints de recourir aux ruses en prétendant dormir ou être absorbés dans une lecture, comme en témoigne ce chômeur de 28 ans : " Je ne lève pas les yeux du livre si je pense que je verrai quelque chose ou quelqu'un à qui je dois céder la place. »

La mise en scène de l'endurance fonctionne comme une preuve du bien-fondé des distinctions opérées entre les hommes et les femmes. Il est donc fort significatif que,

\footnotetext{
${ }^{10}$ Le mot « guerre » fait allusion à ce qu'on appelle en Russie la Grande Guerre nationale (19411945). Même si la majorité des personnes qui ont participé à la victoire contre le nazisme sont déjà décédées, dans l'imaginaire collectif, il persiste l'idée que « le peuple [soviétique] a gagné la guerre » et que cet exploit fait toujours partie des acquis de tout le monde.
} 
lorsqu'il est question de la transmission du devoir de céder la place, les personnes interrogées rappellent volontiers que les garçons sont des «futurs hommes» et qu'ils doivent apprendre à se conduire en conséquence :

Les mamies font souvent l'erreur de laisser les jeunes garçons assis. Elles refusent même de s'asseoir quand un garçon leur cède la place. Après, si c'était un jeune homme [qui cédait la place], elle se serait assise, mais puisqu'il s'agit d'un enfant, qu'il reste assis ce petit, voilà. Hé bien ça, je pense, ça a un impact négatif sur l'éducation. Parce que les garçons doivent céder la place. [...] C'est un élément de... de... du comportement masculin correct... de l'apprentissage d'un certain comportement, voilà. [Petr, enseignant dans le supérieur, 56 ans]

Il est en effet très fréquent que les propositions de place faites par les enfants ne soient pas acceptées, car en dépit de l'obligation de s'entraîner à céder la place, l'enfant est perçu comme encore fragile et vulnérable. Il n'en reste pas moins que si cette proposition est acceptée et qu'elle est faite par un garçon, le geste sera approuvé comme un signe de conformité au "comportement masculin correct». Pour les garçons, apprendre à céder la place s'inscrit donc dans le cadre de l'acquisition d'un «certain comportement» contribuant à la construction de l'identité genrée. Pour la même raison, accepter une proposition de place est fortement déstabilisant pour un homme d'un âge avancé, car cela affiche sa catégorisation non pas comme "homme», mais comme «vieux ». De sorte que si une femme propose sa place à un homme qu'elle ne connaît pas, celui-ci aura du mal à l'accepter. Enfin, une jeune fille assise devant un homme âgé peut trouver plus de raisons pour rester assise en se positionnant, comme «femme en présence de l'homme», que si elle était assise en présence d'une femme âgée, car dans ce cas, elle serait contrainte de jouer le rôle de «jeune ». Se tenir debout, cette « technique du corps » (Mauss, 1993 [1934]) associée au masculin revêt donc toute une distinction sociale, et comme nous le verrons dans la partie suivante, lorsqu'une femme cède sa place, ce ne sont pas les mêmes significations qui entrent en jeu.

\section{L'empathie, un devoir féminin ?}

Même si se tenir debout représente un modèle de comportement exemplaire pour les garçons, les filles apprennent aussi à céder la place. Il s'agit en effet d'un geste aux significations plurielles : outre son inscription dans les rapports de genre, il exprime des égards ou de l'attention, notamment envers les personnes âgées, comme cette séquence en fait état:

Une fille de neuf ans environ est assise à l'extrémité de la banquette. Dès que, près d'elle, se place une femme âgée vêtue d'une veste bordeaux bien soignée, la fille se lève. La femme se penche vers la fille, l'enlace légèrement par les épaules et dit d'une voix très douce et attentionnée: "Merci, merci, ma chérie, mais je vais sortir à la station suivante, alors, assieds-toi, assieds-toi ». La fille se rassoit d'un air légèrement 
désemparé. La femme sort à la station suivante. [Journal de terrain, 07/06/12, Ligne 2]

On voit ici que la femme âgée réagit à l'initiative de la fille avec nuance : elle tâche de refuser sans décourager, par ailleurs, l'enfant à céder la place dans le futur. Même si une fille n'est pas supposée se montrer spécialement forte et résistante, elle est tenue de céder la place dans la mesure où les jeunes se doivent d'être respectueux et compatissants envers les faiblesses de l'âge avancé. C'est ainsi que l'on se montre polie et bien élevée, comme l'explique cette fille de dix ans :

Nina : J'ai cédé la place... à.... à un papy, si je me souviens bien... [...]

Moi : Pourquoi?

Nina: Ben il me semblait que c'était indécent si un adulte est debout et toi tu es assise.

Moi : Et pourquoi penses-tu comme ça?

Nina : Surtout quand c'est une personne âgée, avec une canne, elle se tient à peine debout.

Moi : Oui, ça je comprends, mais ça te vient d'où ? Qui te l'a dit ?

Nina : Ah, mais à l'école, on nous expliquait... des choses... comment monter dans le bus, où il faut traverser la rue, tout ça. [Nina, élève, 10 ans]

Lorsque la conversation touche à la genèse de leurs propres habitudes, les personnes interviewées évoquent souvent leur éducation. La phrase « je cède la place parce que je suis éduqué comme ça » dispense celui qui la prononce de toute autre justification. Dire « je le fais parce que ma maman me l'a appris » ou «c'est ce qu'on nous apprend à l'école », c'est se poser en enfant sage et obéissant, soumis à l'autorité des injonctions parentales ou scolaires. Cependant, on peut remarquer qu'avec une telle justification, la dimension morale de l'offre de sa place devient quelque peu bancale, ce qui ressort bien de l'entretien avec Nina où la règle de céder la place se mélange avec les règles de sécurité routière ( comment monter dans le bus..." ${ }^{11}$ ).

Certes, la démonstration d'une bonne éducation attire un certain prestige social. Or, ce dernier n'est pas le même que celui lié aux conduites connotées comme masculines. Le zèle de l'obéissance ne s'apprend pas de la même manière chez les garçons que chez les filles (Baudelot, Establet, 2007). On peut voir un avatar de cette figure de la fille sage - incarnation par excellence de l'éducation féminine comme l'ont décrite de Beauvoir (1949) et Belotti (1974) - dans l'idée avancée par une étudiante de 25 ans. Expliquant pourquoi, de son point de vue, les femmes cèdent la place plus souvent que les hommes - et cela malgré la double obligation (respect et galanterie) qui est censée engager ces derniers -, elle dit: «Je pense que ce sont les femmes quand même... mais, je ne sais pas pourquoi... c'est notre complexe de la première de la classe, je pense. » Ce propos sous-entend que c'est dans la mesure où l'attente concerne les

\footnotetext{
${ }^{11}$ Sur cette imbrication des différents types de règles dans l'apprentissage des conduites dans les
} milieux urbains, voir Rivière (2018). 
deux sexes que les filles vont avoir tendance à y exceller plus afin de se montrer "premières ».

Et pourtant les femmes éprouvent l'obligation d'exprimer des égards envers les personnes âgées, d'être attentionnées et respectueuses, pour des raisons qui dépassent l'ambition personnelle d'être une «bonne élève». Les attentes envers la gentillesse pèsent sur les femmes plus que sur les hommes. Plusieurs enquêtées témoignent ainsi avoir été l'objet d'une pression ciblée découlant de leur docilité supposée ou bien de l'absence de dispositions à «protester».

Daria: Je peux me rappeler une situation quand on m'a demandé, je ne sais pas pourquoi, de céder la place bien qu'il y ait eu plein de mecs autour, d'un âge où, on dirait, il est approprié de céder la place.

Moi : C'était quoi comme âge?

Daria : Ben, vingt ou vingt-cinq ans. C'est-à-dire qu'il y a un jeune homme dans une bonne forme physique, mais le choix tombe, comme par hasard, sur une jeune fille de quinze ans. Eh ben, de toute évidence, le choix tombe ainsi parce qu'on pense que la jeune fille ne va pas protester. [Daria, étudiante, 26 ans]

Ce témoignage fait apparaître l'usage sélectif de la règle obligeant les jeunes au respect des personnes âgées : interpeller un jeune homme «dans une bonne forme physique » semble être plus dangereux que de s'adresser à une jeune fille. Ce qui prouve qu'en situation la force de l'homme implique non seulement le devoir de protection envers les plus faibles, mais aussi la possibilité de s'extraire - ne serait-ce que ponctuellement - de cette obligation. Le sens de l'action de céder la place se partage ainsi : pour les femmes, cela fait partie des "règles élémentaires de politesse», tandis que, pour les hommes, cela revient à se conduire "en homme». Ce partage donne le ton à l'apprentissage différencié des manières de se comporter et de construire la relation avec autrui : l'attention à l'autre, pour les filles ; l'endurance, pour les garçons. Avancée par certaines personnes interrogées, l'idée que les femmes cèdent la place plus souvent que les hommes parce qu'elles sont "plus attentives aux autres» ou "plus compatissantes» témoigne de la distribution genrée du travail émotionnel (Hochschild, 2003).

Cependant, l'orientation sociale des femmes vers la conformité aux normes ne s'incarne pas nécessairement dans une posture de résignation timide. Les femmes font aussi preuve de conduites concurrentielles et de conquête du prestige, comme on le voit dans l'anecdote suivante:

Une fois, quand j'étais enceinte, j'étais assise et... les banquettes étaient toutes occupées, alors une vieille mamie s'approche et dit: "Voilà une jeune, elle est assise ». Je me suis levée, mais elle a dit tout de suite : « Excusez-moi ». [...] J'avais même envie de me lever, je me demandais comment elle allait réagir. Eh bien, elle a répondu... elle s'est même excusée auprès de moi. Eh bien, même si elle s'était assise, je me serais sentie mieux, parce que j'aurais cédé la place, donc, je me serais montrée à la hauteur... et elle, elle aurait dû avoir honte. [Galina, agent immobilier, 48 ans] 
On voit ici un comportement qui affiche une conformité à la règle du respect des plus âgés, mais qui n'en est pas moins une forme de contestation à leur égard. Tout en prenant la forme d'un sacrifice de son bon droit à rester assise tranquillement en tant qu'une femme enceinte, le geste de Galina a également une autre dimension, plus insidieuse: sa manière de se rendre irréprochable est aussi une manière de mettre en scène sa supériorité, car Galina ne se sent «à la hauteur » que suite aux prétentions hâtives de la « vieille mamie» qui, quant à elle, est contrainte de s'excuser, une fois que l'injustice de sa demande est avérée. Même si elle n'est pas l'initiatrice de l'embarras de la femme âgée, elle en profite bel et bien, se rendant compte qu'elle veut assister au dénouement ( $j$ j'avais même envie de me lever... »). Ce qu'illustre aussi cette anecdote, c'est l'usage stratégique des convenances: la politesse devient une sorte de paravent qui permet à une personne de se protéger tout en exprimant un antagonisme avec l'autre.

\section{4. Éviter le refus lorsqu'une place est proposée}

Le problème de la politesse perfide, celle qui humilie ou rend mal à l'aise, trouve toute son extension dans le cas de l'offre de sa place assise dans le métro. Plusieurs personnes interrogées, et plus particulièrement les femmes, ont témoigné du fait que, dans certains cas, une proposition de place leur avait été vaguement déplaisante. Le désagrément semble provenir principalement du fait que le geste en question met en scène l'asymétrie entre les passagers en suspendant leur parité en tant que quidams. Une idée sous-jacente, qui alimente cette asymétrie, est que dans les transports en commun on ne s'assied pas sans nécessité. Mais qui a besoin de s'asseoir? Dans le registre de la logique catégorielle, ce sont les groupes conçus comme vulnérables, à savoir les personnes âgées ou en situation de handicap, les femmes et les enfants. Leur besoin de s'asseoir est d'emblée associé à une faiblesse enracinée dans la représentation ordinaire de ces personnes comme porteuses de telles ou telles qualités « typiques» (Cefaï, 1994). En ce qui concerne les personnes âgées, c'est justement cette infirmité présumée (" elles doivent ménager leur santé », « elles ont mal aux jambes») qui réunit les gens parvenus à un certain âge dans le groupe "personnes âgées » en impliquant le devoir de protection envers eux. Les jeunes, en revanche, sont censés être forts, à tel point que l'on ne prend souvent pas au sérieux leurs déclarations de fatigue : les personnes plus âgées réagissent souvent à ce type de déclarations comme à quelque chose d'inouï ("Comment? Vous êtes jeunes! ») et la façon acceptable, pour les jeunes, de dire qu'ils sont fatigués et veulent rester assis, se situe dans le registre de la plaisanterie.

D’après certains entretiens, la raison légitime de céder sa place se construit non pas dans la logique catégorielle (tel type de personnes doit être assis), mais dans la logique situationnelle (qu'est-ce qui fait concrètement que cette personne a maintenant plus besoin que moi d'être assise ?). Dans leur proposition de place, au lieu de faire 
référence à l'appartenance aux catégories, ces acteurs font référence aux propriétés situationnelles. Pour convaincre, la prise en compte de l'état concret de l'individu est mise en avant:

Parfois, on se met à refuser : "Non, non, restez ». Dans ce cas, je dis : " Mais enfin, vous avez des sacs tellement lourds, asseyez-vous, donc ». [...] Je ne vais pas lui dire que vous êtes vieille, alors, asseyez-vous, n'est-ce pas ? Ben, d'habitude on cède la place à quelqu'un qui, eh bien, on ne va pas céder la place à une femme qui a, comment dire... quarante ou cinquante ans... mais qui a l'air sportive, est toute énergique, porte une casquette et des tennis. Ça ne vous viendrait même pas à l'esprit qu'il faille lui céder la place. [Véra, enseignante dans le supérieur, 34 ans]

Comme il ressort de ces propos, les acteurs trouvent nécessaire de dissimuler les références à lâge dans leurs interactions. En revanche, reconnaître que la personne porte des sacs lourds, c'est se montrer présent, ouvert à l'autre et attentif aux détails. Le bagage, même s'il provoque souvent le désagrément et les altercations (« retirez votre sac!»), dans le cas de l'offre de la place, est précisément ce qui permet de rendre la proposition opportune et bien fondée. Inversement, un passager peut s'irriter qu'une place assise ne lui ait pas été proposée lorsqu'il porte des sacs lourds et/ou volumineux et que personne ne semble les remarquer. Les objets tels que des béquilles ou une canne jouent le même rôle d'indice matériel laissant entendre que la personne pourrait avoir besoin de s'asseoir. Par ailleurs, d'après les observations et les entretiens, les personnes à qui la place est cédée avec le moins d'hésitation et avec le plus de cordialité partagée par l'expression de la reconnaissance en retour, sont les femmes enceintes. Ces détails suggèrent qu'il est moins risqué de se fier aux états concrets et observables - et parfois transitoires comme la grossesse ou une jambe plâtrée - que de se laisser guider par les considérations plus formelles comme la déférence ou la galanterie. Enfin, la qualification d'une personne âgée comme destinataire des places assises est une affaire d'interprétation: plusieurs acteurs disent traiter l'âge avec souplesse, en prenant en compte l'allure de la personne. D'après Véra, si une femme âgée a l'air « énergique », il ne lui viendra pas à l'esprit qu'elle ait besoin de s'asseoir. Comme l'a exprimé une autre enquêtée, étudiante de 19 ans: "si c'est une mamie robuste et gaillarde [...] je reste assise ».

La configuration semblable se dessine dans les rapports hommes-femmes. Pour une femme, proposer à un homme de s'asseoir constitue un renversement du modèle habituel, et pourtant, dans certaines conditions, l'offre peut finir par être acceptée:

À la station "Gor'kovskaya», parmi les entrants se trouve une femme d'une quarantaine d'années qui porte un sac; il n'est pas très grand, mais apparemment très lourd. La femme le met sur la banquette entre mon genou et le genou de ma voisine, une jeune fille. Celle-ci commence à se lever, mais la femme l'arrête en mettant la main sur son épaule : «Restez, restez, c'est seulement mon sac qui est lourd ». La fille finit par se lever. La femme au sac se retourne, tire la manche d'un homme d'une cinquantaine d'années debout derrière elle et l'invite avec un signe de 
tête à s'asseoir. L'homme sourit et secoue la tête : « Merci, mais cela me gêne... vous êtes une femme quand même ». La femme sourit en retour: " C'est rare, n'est-ce pas ? Mais ça vous fait plaisir... allez... moi je vais sortir ». L'homme: « Moi aussi. Merci. » Pendant cet échange, la femme, tout en souriant, tire le bras de l'homme avec une certaine insistance. II finit par s'asseoir, tombe presque sur le siège. [Journal de terrain, 28/05/12, Ligne 2]

Pour ne pas délaisser l'initiative de la jeune fille qui lui offre sa place, la femme au sac lourd s'engage à installer sur cette place quelqu'un d'autre. Mais puisqu'elle le propose à un homme, ce dernier a du mal à l'accepter. Tout en reconnaissant la singularité de la situation et en laissant entendre qu'elle le fait pour faire plaisir, la femme continue à insister d'une manière qui frôle l'envahissement (on pourrait se demander si son geste serait acceptable si les rôles étaient inversés et si c'était un homme qui tirait la manche d'une femme...). Par ailleurs, elle justifie le fait qu'elle ne peut pas prendre cette place en arguant qu'elle «sor[t] bientôt ». C'est aussi le cas de l'homme, mais il s'assoit quand même: la conduite de la femme lui donne les gages de la bonne foi et finit, manifestement, par porter ses fruits.

À l'inverse, l'offre de la place à une femme tout simplement parce qu'elle est une femme peut être refusée. C'est ce qui semble s'actualiser dans cette séquence, où une jeune femme se voit offrir une place sans raison explicite:

La rame est presque vide. Sur la banquette devant moi sont assis deux jeunes hommes de dix-huit ans environ. Une jeune femme entre, s'arrête devant leur banquette, mais légèrement plus loin d'eux. En face d'elle, une place libre. L'un des jeunes hommes s'adresse à elle en indiquant cette place: "Asseyez-vous!». La femme secoue négativement la tête. II répète d'une façon plus insistante : "Allez, asseyez-vous! », puis ajoute avec un petit sourire : «Sinon, on vous y jette ! ». La femme ne bouge pas, regarde dans la vitre. L'autre jeune homme tient dans ses mains un journal qu'il semble lire. Les deux jeunes hommes échangent quelques répliques indistinctes à propos du journal. Le premier l'enlève à son copain, montre quelque chose sur la page d'un geste si violent, que la page se déchire. II rejette le journal, se dresse, tire le capuchon de sa veste sur la tête, semble troublé. Puis, il se met à embêter son copain, lève le genou en menaçant de le frapper. Celui-ci le repousse en riant. Le jeune homme à la capuche se rassoit, puis fait un petit avion d'une page du journal. La rame freine. Les deux copains s'approchent de la sortie. Au moment de l'ouverture des portes, le jeune homme à la capuche envoie le petit avion qui atterrit aux pieds de la jeune femme. Elle tourne la tête vers la porte, le jeune homme la regarde, brandit le journal roulé d'un geste menaçant, fait une grimace. Puis ils sortent. [Journal de terrain, 31/05/12, Ligne 3]

Comment interpréter l'invitation de s'asseoir dans une situation où l'on se tient debout non pas par nécessité, mais par choix? S'agit-il d'un geste d'aide ou d'un flirt? Apparemment, le jeune homme à la capuche est gêné de voir une femme debout devant lui et essaie d'être aimable, mais il ne peut pas y parvenir de manière classique, en 
cédant sa place, car plusieurs sièges sont inoccupés. Après une invitation à s'asseoir sur une place libre - concise et injonctive, suivie d'un refus tout aussi laconique-, ce jeune homme donne l'impression de ne pas savoir où se mettre. Sa tentative de persuader la jeune femme en la menaçant ( Sinon, on vous y jette!») n'aboutit pas et il se tourne d'abord vers son journal, puis vers son ami. Le refus de la jeune femme semble peser sur lui jusqu'à la fin du voyage, ce qui ressort de son geste avant la sortie. L'embarras suscité par le refus a été plusieurs fois rapporté lors des entretiens: la place refusée est qualifiée de «maudite », à laquelle il est impossible de s'y rasseoir. L'issue de l'action dépend donc en grande partie de la réaction de l'autre. Dans la séquence ci-dessus, la jeune femme considère, probablement, le geste du jeune homme comme malvenu et invasif.

L'offre d'un siège peut apparaître moins rébarbative lorsqu'elle est ancrée non pas dans le répertoire des rôles genrés, mais dans les circonstances. C'est ce dont témoigne Margarita, qui explique pourquoi il lui arrive d'accepter ou de refuser les propositions de place faites par les jeunes hommes:

Ça dépend de la situation, dans quel contexte on cède la place. Lorsque l'on cède d'une telle manière que... visiblement... avec un air condescendant, comme "Voilà, mademoiselle, asseyez-vous ». Mais parfois ça se passe parce que, simplement, bien, disons, j'ai un sac très lourd, j'ai l'ordinateur portable sur moi, il y a un paquet de nourriture et il y a un jeune homme qui voit ça et il se lève comme ça... eh bien, merci, oui. [Margarita, doctorante, 24 ans]

Ici, l'offre est réussite du point de vue interactionnel parce qu'elle évite la catégorisation en neutralisant l'air protecteur et « condescendant ». En effet, la position assise de la femme, avec l'homme debout en surplomb, participe à la ritualisation de la subordination féminine (Goffman, 1977). À cet égard, les chercheurs soulignent la dimension dangereuse de la politesse : puisqu'elle marque objectivement la déférence, elle peut aussi masquer une agression délibérée (Cortès, 2008:57-58) en devenant une « attaque courtoise » (Fracchiolla, Romain, 2015) qui vise à faire assumer à l'autre une place inférieure. Une grande partie du problème politique et moral soulevé par la question de la politesse porte sur les hiérarchies sociales qu'elle peut camoufler en rendant la domination plus acceptable pour les dominés, notamment pour les femmes (Gardner, 1995).

Dans la mesure où le geste a des connotations genrées, il introduit les potentialités d'un jeu de séduction (Gayet-Viaud, 2009). Les entretiens réalisés montrent en effet que l'offre d'un siège peut signifier l'amorce d'un lien interactionnel, susceptible être indésirable aussi bien pour les femmes que pour les hommes. Il peut donc s'avérer périlleux de céder sa place à une personne de sexe opposé. Cela transparaît dans les témoignages de ceux qui disent préférer offrir sa place à une personne âgée plutôt qu’à une jeune femme, car cette dernière situation crée l'impression qu'il s'agit d'une drague: « Je ne cède pas la place aux jeunes femmes, car sinon elles vont penser que je les drague, et je veux pas de ça» (Sémion, traducteur, 25 ans). Néanmoins, certains 
hommes interviewés ont témoigné éprouver du plaisir à céder leur place à « une belle femme» et à se montrer « gentleman ».

\section{Conclusion}

Au terme de cette analyse, il apparaît que la pratique de céder la place dans le métro de Saint-Pétersbourg oscille constamment entre des logiques catégorielle et situationnelle. Dans certains cas, l'offre s'appuie clairement sur le répertoire des rôles traditionnellement attribués aux hommes et aux femmes, aux jeunes et aux personnes âgées. Dans d'autres cas, le geste fait plutôt appel aux circonstances en supposant, en creux, l'égalité des droits sur les places assises. Contrairement aux raisons catégorielles comme l'appartenance à tel ou tel groupe social (qu'il s'agirait encore de bien décrypter...), le besoin de s'asseoir, observable chez l'autre, confère au geste en question une pertinence situationnelle: il ne s'agit pas d'une faiblesse qu'on suppose a priori chez l'autre, mais de ce qui est propre au contexte singulier. Dans ce cadre, le geste évite la problématique de la politesse de surface, d'égards insincères, de la conformité aux règles formelles qui masqueraient une violence potentielle. En revanche, il acquiert la signification d'entraide spontanée et réflexive, dont la dimension morale est en quelque sorte plus convaincante que s'il s'agissait d'une démonstration du respect ou de l'attention, inculquée par la «bonne éducation».

La limite de la logique situationnelle se révèle dans le fait qu'en conditions réelles, il est souvent difficile pour les acteurs d'être certains qu'une autre personne veuille vraiment s'asseoir. C'est pourquoi l'une des façons de désamorcer les complications auxquelles toute relation de face à face peut aboutiri2 c'est de céder la place sans en avoir l'air, en faisant semblant de se préparer à sortir. Une autre astuce consiste à annoncer ouvertement que l'on sort à la prochaine station: cela implique que l'on ne se prive pas, et que céder sa place n'est qu'une manière de s'avancer plus rapidement vers la sortie. Ici, le geste se justifie en s'inscrivant dans le registre de «l'aide restreinte » (restrained helpfulness) (Lofland, 1998), celle qui est de mise entre inconnus et qui n’implique pas nécessairement l'assignation à des catégories.

\section{Bibliographie}

ANDERSON E. (1990), Streetwise: Race, Class, and Change in an Urban Community, Chicago, University of Chicago Press.

AugÉ M. (2009 [1986]), Un ethnologue dans le métro, Paris, Hachette Littérature.

BAUDELOT C., EsTABLET R. (2007), Quoi de neuf chez les filles? Entre stéréotypes et libertés, Paris, Nathan.

\footnotetext{
12 Pour la lecture des normes de civilité en tant que fondées sur l'évitement, voir Goffman (1963
} 1973 [1956] ; 1974 [1967]) et la critique de cette lecture : Gayet-Viaud $(2008 a ; 2009 ; 2011 ; 2019)$ 
Beauvoir S. DE (1949), Le Deuxième Sexe, tome 2 : l'expérience vécue, Paris, Gallimard. BeLotTi E. G. (1974), Du côté des petites filles, Paris, Éditions des femmes.

Bidet A., Boutet M., Chave F., Gayet-Viaud C., Le Méner E. (2015), «Publicité, sollicitation, intervention », SociologieS. En ligne, consulté le 31 mars 2021. URL : http://journals.openedition.org/sociologies/4941.

Birenbaum A., Sagarin E. (dir.) (1973), People in Places: The Sociology of the Familiar, New York, Praeger.

Boltanski L. (1971), « Les usages sociaux du corps », Annales, vol. 26, nº 1, p. 205-233.

BouRdieu P. (1998), La Domination masculine, Paris, Seuil.

Brevigleri M., Trom D. (2003), «Troubles et tensions en milieu urbain. Les épreuves citadines et habitantes de la ville », in D. CEFAÏ, D. PASQUIER (dir.), Les Sens du public. Publics politiques, publics médiatiques, Paris, Presses universitaires de France, p. 399-416.

Carlbäck H., Gradskova Y., Kravchenko Z. (2012), And They Lived Happily Ever After: Norms and Everyday Practices of Family and Parenthood in Russia and Eastern Europe, Budapest, Central European University Press.

CEFAÏ D. (1994), « Type, typicalité, typification. La perspective phénoménologique », in B. FRADIN, L. QUÉRÉ, J. WIDMER (dir.), L'enquête sur les catégories. Raisons pratiques 5 , Paris, Éditions de l'École des Hautes Études en Sciences Sociales, p. 105-128.

CEFAÏ D. (2013), «L'ordre public. Micropolitique de Goffman », postface à GofFMAn E. (2013 [1963]), Comment se conduire dans les lieux publics. Notes sur l'organisation sociale des rassemblements, Paris, Éditions Economica, p. 207-288.

CORTĖS J. (2008), « "Mouche ton nez, dis bonjour à la dame !" Réflexions didactologiques sur la politesse et sur sa vision spéculaire », Synergies Espagne, nº 1, p.47-58.

Darmon M. (2003), Devenir anorexique, Paris, La Découverte.

DuNEIER M. (1999), Sidewalk, New York, Farrar, Strauss and Giroux.

FracCHIOlla B., Romain C. (2015), «L'attaque courtoise: un modèle d'interaction pragmatique au service de la prise de pouvoir en politique», Semen, $n^{\circ} 40$. En ligne, consulté le 11 mars 2020. URL: http://journals.openedition.org/semen/10418.

GARDNER C. B. (1995), Passing By. Gender and Public Harassment, Berkeley, University of California Press.

GAYET-VIAUD C. (2008a), L'Égard et la règle. Déboires et bonheurs de la civilité urbaine, thèse de doctorat, Paris, École des Hautes Études en Sciences Sociales.

GAYET-VIAUD C. (2008b), «Les disputes de politesse dans l'espace urbain. Quand la politesse tourne à la violence», in C. MoḮs, C. SCHULTZ-Romain (dir.), De l'impolitesse à la violence verbale, Paris, L'Harmattan, p. 147-183.

GAYET-VIAUD C. (2009), « La femme autonome et l'homme galant. Intransigeances du respect et acrobaties de l'égard dans la civilité urbaine», in M. JouAN, S. LAUGIER (dir.), Penser l'autonomie, Paris, Presses universitaires de France, p. 389-406. 
GAYET-VIAUD C. (2011), «La moindre des choses. Enquête sur la civilité urbaine et ses péripéties », in M. BERGER, D. CEFAÏ, C. GAYET-VIAUd (dir.), Du civil au politique: ethnographies du vivre-ensemble, Bruxelles, PIE Peter Lang, p. 27-54.

GAYET-VIAUD C. (2019), « La civilité est-elle réac ? », Politix, vol. 125, n 1, p. 31-58.

GoffMAN E. (1963), Behavior in Public Places. Notes on the Social Organization of Gatherings, New York, Free Press (traduction française de D. CEFAII sous le titre Comment se conduire dans les lieux publics. Notes sur l'organisation sociale des rassemblements, Paris, Éditions Economica, 2013).

Goffman E. (1973 [1956]), La Mise en scène de la vie quotidienne, tome 1: La Présentation de soi, Paris, Éditions de Minuit.

GofFMAN E. (1974 [1967]), Les Rites d'interaction, Paris, Éditions de Minuit.

GoffMAN E. (1977), «La ritualisation de la féminité», Actes de la recherche en sciences sociales, vol. 14, p. 34-50.

Goffman E. (2002 [1977]), L’Arrangement des sexes, Paris, La Dispute.

HAICAULT M. (1993), « La Doxa de sexe, une approche du symbolique dans les rapports sociaux de sexe », Recherches féministes, vol. 6, n 2, p. 7-20.

HoCHSCHILD A. R. (2003), « Travail émotionnel, règles de sentiment et structure sociale», Travailler, vol. 9, $\mathrm{n}^{\circ} 1, \mathrm{p} .19-49$.

Joseph I. (1984), Le Passant considérable: essai sur la dispersion de l'espace public, Paris, Librairie des méridiens.

JOSEPH I. (2007), L'Athlète moral et l'enquêteur modeste, Paris, Éditions Economica.

Kelly C. (2001), Refining Russia: Advice Literature, Polite Culture, and Gender from Catherine to Yeltsin, New York, Oxford University Press.

LEE J. R. E., WATSON R. (1992), « Regards et habitudes des passants: Les arrangements de visibilité de la locomotion », Les Annales de la recherche urbaine, n 57-58, p. 101-109.

LeVINE J., Vinson A., WoOD D. (1973), «Subway Behavior », in A. BiRENBAUM, E. SAGARIN (dir.) (1973), People in Places: The Sociology of the Familiar, New York, Praeger, p. 208217.

LIEBOW E. (2011 [1967]), Tally's Corner. Les Noirs du coin de la rue, Rennes, PUR.

LOFLAND L. H. (1973), A World of Strangers: Order and Action in Urban Public Space, New York, Basic Books.

LOFLAND L. H. (1998), The Public Realm: Exploring the City's Quintessential Social Territory, Hawthorne/New York, Aldine de Gruyter.

MAuss M. (1993 [1934]), « Notion de technique du corps», in M. MAuss, Sociologie et anthropologie, Paris, Presses universitaires de France, p. 365-389.

Nielsen L. B. (2004), License to Harass: Law, Hierarchy, and Offensive Public Speech, Princeton, Princeton University Press.

QUÉRÉ L., BREZGER D. (1992), « L'étrangeté mutuelle des passants : Le mode de coexistence du public urbain », Les Annales de la recherche urbaine, $n^{\circ}$ 57-58, p. 89-100.

RIVIÈRE C. (2018), « Entre méfiance, prudence et politesse : quand les parents enseignent à leurs enfants comment se conduire dans les espaces publics urbains à Paris et 
Milan », Enfances Familles Générations, vol. 30. En ligne, consulté le 03 février 2021. URL: http://journals.openedition.org/efg/2484.

RyaVe A. L., SCHENKeIN J. N. (1974), « Notes on the Art of Walking », in R. TuRnER (dir.), Ethnomethodology, Harmondsworth, Penguin, p. 265-274.

SACKS H. (1979), «Hotrodder: A Revolutionary New Category», in G. PSATHAS (dir.), Everyday Language: Studies in Ethnomethodology, New York, Irvington, p. 7-14.

SCHWARTZ B. (1975), Queuing and Waiting: Studies in the Social Organization of Access and Delay, Chicago, The University of Chicago Press.

SENNETT R. (1979 [1977]), Les Tyrannies de l'intimité, Paris, Seuil.

SuDNOw D. (1972), «Temporal Parameters of Interpersonal Observation », in D. SuDNOW (dir.), Studies in Social Interaction, New York, Free Press, p. 259-279.

TAVORY I. (2011), « À la vue d'une kippa. Une phénoménologie des attentes d'interaction dans un quartier juif orthodoxe de Los Angeles », in M. BERGER, D. CEFAÏ, C. GAYETVIAUD (dir.), Du civil au politique: ethnographies du vivre-ensemble, Bruxelles, PIE Peter Lang, p. 55-76.

TONNELAT S. (2012), «Confiance et émotions dans le métro de New York », in E. LE MOUËL, C. EspinasSe (dir.), Lieux et Liens, tome 1: Des lieux qui créent des liens, Paris, L'Harmattan, p. 201-228.

VAKHTIN N., FIRSOV B. (dir.) (2017), «Sindrom publichnoy nemoty ». Istoria i sovremennye praktiki publichnih depatov v Rossii [ Syndrome du mutisme en public ». L'histoire et les pratiques contemporaines des débats publics en Russiel, Moscou, Novoe Literaturnoe Obosrenie.

Weilenmann A., NoRmark D., LAURIER E. (2014), « Managing Walking Together: The Challenge of Revolving Doors », Space and Culture, vol.17, n 2, p.122-136.

WOLFF M. (1973), « Notes on the behaviour of pedestrians », in A. BIRENBAUM, E. SAGARIN (dir.), People in Places: The Sociology of the Familiar, New York, Praeger, p. 35-48.

ZdRAVOMYsLOVA E., RotKIRCH A., TEMKINA A. (dir.) (2009), Novyi byt $v$ sovremennoi Rossii: gendernye issledovania povsednevnosti [Nouveau mode de vie dans la Russie contemporaine: études du quotidien sous le prisme du genre], Saint-Pétersbourg, Éditions de L’Université Européenne. 